\title{
On the Adams-Riemann-Roch theorem in positive characteristic
}

\author{
Richard PINK* and Damian RÖSSLER ${ }^{\dagger}$ \\ with an appendix by B. Köck
}

October 24, 2018

\begin{abstract}
We give a new proof of the Adams-Riemann-Roch theorem for a smooth projective morphism $X \rightarrow Y$, in the situation where $Y$ is a regular scheme, which is quasi-projective over $\mathbb{F}_{p}$. We also partially answer a question of B. Köck.
\end{abstract}

\section{Introduction}

Let $Y$ be a regular quasi-projective scheme over an affine finite-dimensional noetherian scheme $S$. Let $X$ be a regular scheme and let $f: X \rightarrow Y$ be a projective morphism of schemes. Let $k \geqslant 2$ be a natural number and $E$ an element of $K_{0}(X)$. The Adams-Riemann-Roch theorem asserts that

$$
\psi^{k}\left(R^{\bullet} f_{*}(E)\right)=R^{\bullet} f_{*}\left(\theta^{k}\left(L_{f}\right)^{-1} \otimes \psi^{k}(E)\right)
$$

in $K_{0}(Y)\left[\frac{1}{k}\right]:=K_{0}(Y) \otimes_{\mathbb{Z}} \mathbb{Z}\left[\frac{1}{k}\right]$. The various symbols appearing in this formula are defined as follows.

The Grothendieck group of locally free coherent sheaves on a scheme $Z$ is denoted by $K_{0}(Z)$, and the Grothendieck group of coherent sheaves on $Z$ is denoted

*Department of Mathematics, ETH Zürich, 8092 Zürich, SWITZERLAND

†Département de Mathématiques, Bâtiment 425, Faculté des Sciences d'Orsay, Université Paris-Sud, 91405 Orsay Cedex, FRANCE 
by $K_{0}^{\prime}(Z)$. The obvious group morphism $K_{0}(Z) \rightarrow K_{0}^{\prime}(Z)$ is an isomorphism whenever $Z$ is regular, noetherian and carries an ample invertible sheaf (see [8, Th. I.9]). In particular it is an isomorphism in the case $Z=Y$. For simplicity we will denote the class in $K_{0}(Z)$ of a sheaf $E$ again by $E$.

For $f: X \rightarrow Y$ as above there is a unique group morphism $K_{0}(X) \rightarrow K_{0}^{\prime}(Y)$ which sends the class of a locally free coherent sheaf $E$ on $X$ to the class of the linear combination $\sum_{j \geqslant 0}(-1)^{j} R^{j} f_{*}(E)$ of coherent sheaves on $Y$. The composite of this group morphism with the inverse of the isomorphism $K_{0}(Y) \stackrel{\sim}{\rightarrow} K_{0}^{\prime}(Y)$ is a group morphism $R^{\bullet} f_{*}: K_{0}(X) \rightarrow K_{0}(Y)$.

To define the symbol $\psi^{k}$, recall that the tensor product of $\mathcal{O}_{Z}$-modules makes the group $K_{0}(Z)$ into a commutative unitary ring and that the inverse image of coherent sheaves under any morphism of schemes $Z^{\prime} \rightarrow Z$ induces a morphism of unitary rings $K_{0}(Z) \rightarrow K_{0}\left(Z^{\prime}\right)$ (see [8, Par. 1]). Thus $K_{0}(\cdot)$ may be viewed as a contravariant functor from the category of schemes to the category of commutative unitary rings. The symbol $\psi^{k}$ refers to an endomorphism of this functor (sic!) that is uniquely determined by the further property that

$$
\psi^{k}(L)=L^{\otimes k}
$$

for any invertible sheaf $L$ (see [8, Par. 16]).

The symbol $\theta^{k}$ refers to a different operation associating an element of $K_{0}(Z)$ to any locally free coherent sheaf on $Z$. It is uniquely determined by the properties:

(i) for any invertible sheaf $L$ on $Z$ we have

$$
\theta^{k}(L)=1+L+\cdots+L^{k-1}
$$

(ii) for any short exact sequence $0 \rightarrow E^{\prime} \rightarrow E \rightarrow E^{\prime \prime} \rightarrow 0$ of locally free coherent sheaves on $Z$ we have

$$
\theta^{k}(E)=\theta^{k}\left(E^{\prime}\right) \otimes \theta^{k}\left(E^{\prime \prime}\right)
$$

(iii) for any morphism of schemes $g: Z^{\prime} \rightarrow Z$ and any locally free coherent sheaf $E$ on $Z$ we have

$$
g^{*}\left(\theta^{k}(E)\right)=\theta^{k}\left(g^{*}(E)\right)
$$


If $Z$ is quasi-projective over an affine finite-dimensional noetherian scheme, it is known that $\theta^{k}(E)$ is invertible in $K_{0}(Z)\left[\frac{1}{k}\right]$ for every locally free coherent sheaf $E$ on $Z$ (see [6, Lemma 4.3]). In that case $\theta^{k}$ extends to a unique map $K_{0}(Z) \rightarrow$ $K_{0}(Z)\left[\frac{1}{k}\right]$ satisfying

$$
\theta^{k}(E)=\theta^{k}\left(E^{\prime}\right) \cdot \theta^{k}\left(E^{\prime \prime}\right)
$$

whenever $E=E^{\prime}+E^{\prime \prime}$ in $K_{0}(Z)$.

The symbol $L_{f}$ denotes the relative cotangent complex of the morphism $f$ : $X \rightarrow Y$. Since $f$ is by construction a local complete intersection morphism, its cotangent complex can be represented by a bounded complex of coherent sheaves (see for instance [3, Illusie, chap. 8, App. G., 8.5.29]). Thus $L_{f}$ determines a unique element of $K_{0}^{\prime}(X)=K_{0}(X)$, for which $\theta^{k}\left(L_{f}\right) \in K_{0}(X)\left[\frac{1}{k}\right]$ is well-defined by additivity. For example, if $f$ is smooth, then $\theta^{k}\left(L_{f}\right)=\theta^{k}\left(\Omega_{f}\right)$.

This explains all the ingredients of the formula (11).

The formula (1) is classically proven using deformation to the normal cone and considering closed immersions and relative projective spaces separately (see [1]).

Our aim in this text is to provide a new and more direct proof of the formula (11) in the specific situation where $k$ is a prime number $p, f$ is smooth and $S$ is a scheme of characteristic $p$, which is of finite type over the finite field $\mathbb{F}_{p}$.

The search for this proof was motivated by the fact that for any scheme $Z$ of characteristic $p$, the endomorphism $\psi^{p}: K_{0}(Z) \rightarrow K_{0}(Z)$ coincides with the endomorphism $F_{Z}^{*}: K_{0}(Z) \rightarrow K_{0}(Z)$ induced by pullback by the absolute Frobenius endomorphism $F_{Z}: Z \rightarrow Z$. This is a consequence of the splitting principle [8, Par. 5]. We asked ourselves whether in this case $\theta^{p}\left(L_{f}\right)=\theta^{p}\left(\Omega_{f}\right)$ can also be represented by an explicit virtual bundle. If such a representative were available, one might try to give a direct proof of (1i) that does not involve factorisation. The proof given in Section 3 shows that this is indeed possible.

In the article [5, sec. 5] by B. Köck, a different line of speculation lead to a question (Question 5.2) in the context of a characteristic $p$ interpretation of the Adams-Riemann-Roch formula. Our Proposition 2.6 and Proposition 3.2 show that the answer to this question is positive for a large class of morphisms. See the end of section 3 for details.

Fix $k \geqslant 2$ and suppose that $Y$ is the spectrum of a finite field. The formula (1) then formally implies the Hirzebruch-Riemann-Roch theorem for $X$ over that 
field. This is explained for instance in [9, Intro.]. On the other hand, a specialization argument shows that the Hirzebruch-Riemann-Roch theorem for varieties over any field follows from the Hirzebruch-Riemann-Roch theorem for varieties over finite fields. Thus by reduction modulo primes our proof of (1) in positive characteristic leads to a proof of the Hirzebruch-Riemann-Roch formula in general.

The structure of the article is the following. In Section 2, we construct a canonical bundle representative for the element $\theta^{p}(E)$ for any locally free coherent sheaf $E$ on a scheme of characteristic $p$. In Section [3, we give the computation proving (11) in the situation where $k=p, f$ is smooth and $S$ is a scheme of characteristic $p$, which is of finite type over $\mathbb{F}_{p}$.

\section{A bundle representative for $\theta^{p}(E)$}

Let $p$ be a prime number and $Z$ a scheme of characteristic $p$. Let $E$ be a locally free coherent sheaf on $Z$. For any integer $k \geqslant 0$ let $\operatorname{Sym}^{k}(E)$ denote the $k$-th symmetric power of $E$. Then

$$
\operatorname{Sym}(E):=\bigoplus_{k \geqslant 0} \operatorname{Sym}^{k}(E)
$$

is a quasi-coherent graded $\mathcal{O}_{Z}$-algebra, called the symmetric algebra of $E$. Let $\mathcal{J}_{E}$ denote the graded sheaf of ideals of $\operatorname{Sym}(E)$ that is locally generated by the sections $e^{p}$ of $\operatorname{Sym}^{p}(E)$ for all sections $e$ of $E$, and set

$$
\tau(E):=\operatorname{Sym}(E) / \mathcal{J}_{E}
$$

Locally this construction means the following. Consider an open subset $U \subset Z$ such that $E \mid U$ is free, and choose a basis $e_{1}, \ldots, e_{r}$. Then $\operatorname{Sym}(E) \mid U$ is the polynomial algebra over $\mathcal{O}_{Z}$ in the variables $e_{1}, \ldots, e_{r}$. Since $Z$ has characteristic $p$, for any open subset $V \subset U$ and any sections $a_{1}, \ldots, a_{r} \in \mathcal{O}_{Z}(V)$ we have

$$
\left(a_{1} e_{1}+\ldots+a_{r} e_{r}\right)^{p}=a_{1}^{p} e_{1}^{p}+\ldots+a_{r}^{p} e_{r}^{p} .
$$

It follows that $\mathcal{J}_{E} \mid U$ is the sheaf of ideals of $\operatorname{Sym}(E) \mid U$ that is generated by $e_{1}^{p}, \ldots, e_{r}^{p}$. Clearly that description is independent of the choice of basis and compatible with localization; hence it can be used to an equivalent definition of $\mathcal{J}_{E}$ and $\tau(E)$. 
The local description also implies that $\tau(E) \mid U$ is free over $\mathcal{O}_{Z} \mid U$ with basis the images of the monomials $e_{1}^{i_{1}} \cdots e_{r}^{i_{r}}$ for all choices of exponents $0 \leqslant i_{j}<p$. From this we deduce:

Lemma 2.1. If $E$ is a locally free coherent sheaf of rank $r$, then $\tau(E)$ is a locally free coherent sheaf of rank $p^{r}$.

Now we go through the different properties that characterize the operation $\theta^{p}$.

Lemma 2.2. For any invertible sheaf $L$ on $Z$ we have

$$
\tau(L) \cong \mathcal{O}_{Z} \oplus L \oplus \cdots \oplus L^{\otimes(p-1)} .
$$

Proof. In this case the local description shows that $\mathcal{J}_{L}$ is the sheaf of ideals of $\operatorname{Sym}(L)$ that is generated by $\operatorname{Sym}^{p}(L)=L^{\otimes p}$. The lemma follows at once.

Lemma 2.3. For any morphism of schemes $g: Z^{\prime} \rightarrow Z$ and any locally free coherent sheaf $E$ on $Z$ we have

$$
g^{*}(\tau(E)) \cong \tau\left(g^{*}(E)\right) .
$$

Proof. Direct consequence of the construction.

Lemma 2.4. For any two locally free coherent sheaves $E^{\prime}$ and $E^{\prime \prime}$ on $Z$ we have

$$
\tau\left(E^{\prime} \oplus E^{\prime \prime}\right) \cong \tau\left(E^{\prime}\right) \otimes \tau\left(E^{\prime \prime}\right)
$$

Proof. The homomorphism of sheaves

$$
E^{\prime} \oplus E^{\prime \prime} \hookrightarrow \operatorname{Sym}\left(E^{\prime}\right) \otimes \operatorname{Sym}\left(E^{\prime \prime}\right),\left(e^{\prime}, e^{\prime \prime}\right) \mapsto e^{\prime} \otimes 1+1 \otimes e^{\prime \prime}
$$

induces an algebra isomorphism

$$
\operatorname{Sym}\left(E^{\prime} \oplus E^{\prime \prime}\right) \rightarrow \operatorname{Sym}\left(E^{\prime}\right) \otimes \operatorname{Sym}\left(E^{\prime \prime}\right) .
$$

The local description as polynomial rings in terms of bases of $E^{\prime} \mid U$ and $E^{\prime \prime} \mid U$ shows that this is an isomorphism of sheaves of $\mathcal{O}_{Z}$-algebras. Since

$$
\left(e^{\prime} \otimes 1+1 \otimes e^{\prime \prime}\right)^{p}=e^{\prime p} \otimes 1+1 \otimes e^{\prime \prime p}
$$

for any local sections $e^{\prime}$ of $E^{\prime}$ and $e^{\prime \prime}$ of $E^{\prime \prime}$, this isomorphism induces an isomorphism of sheaves of ideals

$$
\mathcal{J}_{E^{\prime} \oplus E^{\prime \prime}} \rightarrow \mathcal{J}_{E^{\prime}} \otimes \operatorname{Sym}\left(E^{\prime \prime}\right) \oplus \operatorname{Sym}\left(E^{\prime}\right) \otimes \mathcal{J}_{E^{\prime \prime}}
$$

The lemma follows from this by taking quotients. 
Lemma 2.5. For any short exact sequence $0 \rightarrow E^{\prime} \rightarrow E \rightarrow E^{\prime \prime} \rightarrow 0$ of locally free coherent sheaves on a noetherian scheme $Z$ we have

$$
\tau(E)=\tau\left(E^{\prime}\right) \otimes \tau\left(E^{\prime \prime}\right)
$$

in $K_{0}(Z)$.

Proof. Let $\widetilde{E}^{\prime}$ and $\widetilde{E}^{\prime \prime}$ denote the inverse images of $E^{\prime}$ and $E^{\prime \prime}$ under the projection morphism $Z \times \mathbf{P}^{1} \rightarrow Z$. Then there exists a short exact sequence

$$
0 \rightarrow \widetilde{E}^{\prime} \rightarrow \widetilde{E} \rightarrow \widetilde{E}^{\prime \prime} \rightarrow 0
$$

of locally free coherent sheaves on $Z \times \mathbf{P}^{1}$ whose restriction to the fiber above $0 \in \mathbf{P}^{1}$ is the given one and whose restriction to the fiber above $\infty \in \mathbf{P}^{1}$ is split (the construction is given in [2, I, Par. f)]). Thus the respective restrictions satisfy $\widetilde{E}_{0} \cong E$ and $\widetilde{E}_{\infty} \cong E^{\prime} \oplus E^{\prime \prime}$. Using Lemmata 2.3 and 2.4 this implies that

$$
\tau(E) \cong \tau\left(\widetilde{E}_{0}\right) \cong \tau(\widetilde{E})_{0}
$$

and

$$
\tau\left(E^{\prime}\right) \otimes \tau\left(E^{\prime \prime}\right) \cong \tau\left(E^{\prime} \oplus E^{\prime \prime}\right) \cong \tau\left(\widetilde{E}_{\infty}\right) \cong \tau(\widetilde{E})_{\infty}
$$

But the fact that $K_{0}\left(Z \times \mathbf{P}^{1}\right)$ is generated by the powers of $\mathcal{O}(1)$ over $K_{0}(Z)$ (see [8, Par. 5]) implies that the restriction to 0 and $\infty$ induce the same map $K_{0}\left(Z \times \mathbf{P}^{1}\right) \rightarrow K_{0}(Z)$. Thus it follows that $\tau(\widetilde{E})_{0}=\tau(\widetilde{E})_{\infty}$ in $K_{0}(Z)$, whence the lemma.

Remark. Lemma 2.5 can also be proved by an explicit calculation of sheaves. For a sketch consider the decreasing filtration of $\operatorname{Sym}(E)$ by the graded ideals $\operatorname{Sym}^{i}\left(E^{\prime}\right) \cdot \operatorname{Sym}(E)$ for all $i \geqslant 0$. One first shows that the associated bi-graded algebra is isomorphic to $\operatorname{Sym}\left(E^{\prime}\right) \otimes \operatorname{Sym}\left(E^{\prime \prime}\right)$. The filtration of $\operatorname{Sym}(E)$ also induces a filtration of $\tau(E)$ by graded ideals, whose associated bi-graded algebra is therefore a quotient to $\operatorname{Sym}\left(E^{\prime}\right) \otimes \operatorname{Sym}\left(E^{\prime \prime}\right)$. To prove that this quotient is isomorphic to $\tau\left(E^{\prime}\right) \otimes \tau\left(E^{\prime \prime}\right)$ one shows that the kernel of the quotient morphism $\operatorname{Sym}\left(E^{\prime}\right) \otimes \operatorname{Sym}\left(E^{\prime \prime}\right) \rightarrow \operatorname{Gr}(\tau(E))$ is precisely $\mathcal{J}_{E^{\prime}} \otimes \operatorname{Sym}\left(E^{\prime \prime}\right) \oplus \operatorname{Sym}\left(E^{\prime}\right) \otimes \mathcal{J}_{E^{\prime \prime}}$. But this is a purely local assertion, for which one can assume that the exact sequence splits. The calculation then becomes straightforward, as in Lemma 2.4, Proposition 2.6. For any locally free coherent sheaf $E$ on a noetherian scheme $Z$ we have $\tau(E)=\theta^{p}(E)$ in $K_{0}(Z)$.

Proof. Combination of Lemmata 2.2, 2.3, 2.5 and the defining properties (i), (ii), (iii) of $\theta^{p}(\cdot)$ in Section 1 . 


\section{Proof of the Adams-Riemann-Roch formula}

Let us now consider the morphism $f: X \rightarrow Y$ of the introduction. Recall that $Y$ is regular and quasi-projective over an affine noetherian finite-dimensional scheme $S$ and that $f$ is projective. We make the supplementary hypothesis that $f$ is smooth and that $S$ is a scheme of characteristic $p$, which is of finite type over the finite field $\mathbb{F}_{p}$. To prove the formula (1) we may also suppose that $Y$ and $X$ are connected and thus integral. Then $f$ has constant fibre dimension, say $r$.

Consider the commutative diagram

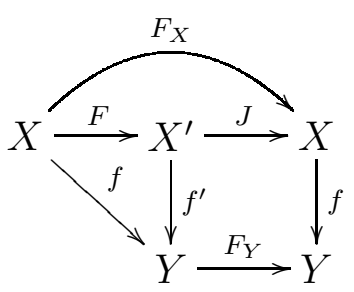

where $F_{X}$ and $F_{Y}$ are the respective absolute Frobenius morphisms and the square is cartesian. The morphism $F=F_{X / X^{\prime}}$ is called the relative Frobenius morphism of $X$ over $Y$. The following lemma summarizes the properties of $F$ that we shall need. For its proof, see [7, Th. 15.7].

Lemma 3.1. The morphism $F$ is finite and flat of constant degree $p^{r}$.

Let $I$ denote the kernel of the natural morphism of $\mathcal{O}_{X}$-algebras $F^{*} F_{*} \mathcal{O}_{X} \rightarrow \mathcal{O}_{X}$, which by construction is a sheaf of ideals of $F^{*} F_{*} \mathcal{O}_{X}$. Let

$$
\operatorname{Gr}\left(F^{*} F_{*} \mathcal{O}_{X}\right):=\bigoplus_{k \geqslant 0} I^{k} / I^{k+1}
$$

denote the associated graded sheaf of $\mathcal{O}_{X}$-algebras. Let $\Omega_{f}$ denote the relative sheaf of differentials of $f$.

Proposition 3.2. There is a natural isomorphism of $\mathcal{O}_{X}$-modules

$$
I / I^{2} \cong \Omega_{f}
$$

and a natural isomorphism of graded $\mathcal{O}_{X}$-algebras

$$
\tau\left(I / I^{2}\right) \cong \operatorname{Gr}\left(F^{*} F_{*} \mathcal{O}_{X}\right) .
$$


Proof. Since $F$ is affine (see Lemma 3.1), there is a canonical isomorphism

$$
\text { Spec } F^{*} F_{*} \mathcal{O}_{X} \cong X \times_{X^{\prime}} X
$$

for which the natural morphism of $\mathcal{O}_{X}$-algebras $F^{*} F_{*} \mathcal{O}_{X} \rightarrow \mathcal{O}_{X}$ corresponds to the diagonal embedding $X \hookrightarrow X \times_{X^{\prime}} X$. We carry out these identifications throughout the remainder of this proof. Then $I$ is the sheaf of ideals of the diagonal, and so $I / I^{2}$ is naturally isomorphic to the relative sheaf of differentials $\Omega_{F}$. On the other hand we have $F^{*} \Omega_{f^{\prime}}=F^{*} J^{*} \Omega_{f}=F_{X}^{*} \Omega_{f}$, which yields a natural exact sequence

$$
F_{X}^{*} \Omega_{f} \rightarrow \Omega_{f} \rightarrow \Omega_{F} \rightarrow 0 .
$$

Here the leftmost arrow sends any differential $d x$ to $d\left(x^{p}\right)=p \cdot x^{p-1} \cdot d x=0$. Thus the exact sequence yields an isomorphism $\Omega_{f} \cong \Omega_{F} \cong I / I^{2}$, proving the first assertion.

For the second assertion observe that, by the universal property of the symmetric algebra $\operatorname{Sym}(\cdot)$, the embedding $I / I^{2} \hookrightarrow \operatorname{Gr}\left(F^{*} F_{*} \mathcal{O}_{X}\right)$ extends to a unique morphism of $\mathcal{O}_{X}$-algebras

$$
\rho: \operatorname{Sym}\left(I / I^{2}\right) \rightarrow \operatorname{Gr}\left(F^{*} F_{*} \mathcal{O}_{X}\right)
$$

We want to compare the kernel of $\rho$ with $\mathcal{J}_{I / I^{2}}$. For this recall that $I$, as the sheaf of ideals of the diagonal, is generated by the sections $s \otimes 1-1 \otimes s$ for all local sections $s$ of $\mathcal{O}_{X}$. The $p$-th power of any such section is

$$
(s \otimes 1-1 \otimes s)^{p}=s^{p} \otimes 1-1 \otimes s^{p}=0
$$

in $F^{*} F_{*} \mathcal{O}_{X}$, because $s^{p}=F_{X}^{*} s$ is the pullback via $F_{X}$ of a section of $\mathcal{O}_{X}$ and hence also the pullback via $F$ of a section of $\mathcal{O}_{X^{\prime}}$. Thus $\rho$ sends the $p$-th powers of certain local generators of $I / I^{2}$ to zero. But in Section 2 we have seen that $\mathcal{J}_{I / I^{2}}$ is locally generated by the $p$-th powers of any local generators of $I / I^{2}$. Therefore $\rho\left(\mathcal{J}_{I / I^{2}}\right)=0$, and so $\rho$ factors through a morphism of $\mathcal{O}_{X}$-algebras

$$
\bar{\rho}: \tau\left(I / I^{2}\right) \rightarrow \operatorname{Gr}\left(F^{*} F_{*} \mathcal{O}_{X}\right) .
$$

From the definition of $\operatorname{Gr}\left(F^{*} F_{*} \mathcal{O}_{X}\right)$ we see that $\rho$ and hence $\bar{\rho}$ is surjective.

On the other hand the smoothness assumption on $f$ implies that $I / I^{2} \cong \Omega_{f}$ is locally free of rank $r$. Thus Lemma 2.1 shows that $\tau\left(I / I^{2}\right)$ is locally free of rank $p^{r}$. By Lemma 3.1 the same is true for $F^{*} F_{*} \mathcal{O}_{X}$ and hence, since $X$ is 
integral, for $\operatorname{Gr}\left(F^{*} F_{*} \mathcal{O}_{X}\right)$ at the generic point $\eta$ of $X$. As $\bar{\rho}$ is surjective, it is therefore an isomorphism at $\eta$. Therefore the sheaf $\operatorname{ker}(\bar{\rho})$ vanishes at $\eta$. But since $X$ is integral, any torsion subsheaf of a locally free coherent sheaf on $X$ is zero. Thus $\operatorname{ker}(\bar{\rho})=0$ everywhere, and so $\bar{\rho}$ is the desired isomorphism.

Remark. The assumption that $f$ is projective was not used in the proof of Proposition 3.2. In particular, its conclusion is valid without this assumption.

Lemma 3.3. Let $Z$ be a quasi-projective scheme of finite dimension over an affine noetherian scheme. Let $E$ be a locally free coherent sheaf of rank $r$ on $Z$. Then the class of $E$ is invertible in the ring $K_{0}(Z)\left[\frac{1}{r}\right]$.

Proof. Let $F^{1} K_{0}(Z)$ be the kernel of the rank morphism $K_{0}(Z) \rightarrow \mathbb{Z}$. This is an ideal whose $k$-th power vanishes for all $k>\operatorname{dim}(Z)$ (for this see [4, V, par. 3, Cor. 3.10]). The infinite sum in $K_{0}(Z)\left[\frac{1}{r}\right]$

$$
1 / r+(r-E) / r^{2}+(r-E)^{\otimes 2} / r^{3}+\ldots
$$

therefore only has a finite number of non-vanishing terms. A direct calculation with geometric series shows that this sum is an inverse of $E$ in $K_{0}(Z)\left[\frac{1}{r}\right]$.

Remark. In [5, Question 5.2], B. Köck in particular asks the following question: is the equation

$$
F_{*}\left(\theta^{p}\left(\Omega_{f}\right)^{-1}\right)=1
$$

valid in $K_{0}(Y)\left[\frac{1}{p}\right]$ ? Proposition 3.2 implies that the answer to this question is positive. Indeed, using the projection formula in $K_{0}$-theory, we compute

$$
F_{*}\left(\theta^{p}\left(\Omega_{g}\right)^{-1}\right)=F_{*}\left(\left(F^{*} F_{*} \mathcal{O}_{Z}\right)^{-1}\right)=F_{*}\left(F^{*}\left(F_{*} \mathcal{O}_{Z}\right)^{-1}\right)=\left(F_{*} \mathcal{O}_{Z}\right) \otimes\left(F_{*} \mathcal{O}_{Z}\right)^{-1}=1
$$

This computation is partially repeated below.

We now come to the proof of the Adams-Riemann-Roch formula, which results from the following calculation in $K_{0}(X)\left[\frac{1}{p}\right]$. This calculation is in essence already in [5, Prop. 5.5]. It did not lead to a proof of the formula (11) there, because the 
Proposition 3.2 was missing.

$$
\begin{aligned}
\psi^{p}\left(R^{\bullet} f_{*}(E)\right) & =F_{Y}^{*} R^{\bullet} f_{*}(E) \\
& =R^{\bullet} f_{*}^{\prime}\left(J^{*}(E)\right) \\
& =R^{\bullet} f_{*}^{\prime}\left(\left(F_{*} \mathcal{O}_{X}\right) \otimes\left(F_{*} \mathcal{O}_{X}\right)^{-1} \otimes J^{*}(E)\right) \\
& =R^{\bullet} f_{*}^{\prime} F_{*}\left(F^{*}\left(F_{*} \mathcal{O}_{X}\right)^{-1} \otimes F^{*} J^{*}(E)\right) \\
& =R^{\bullet} f_{*}\left(\left(F^{*} F_{*} \mathcal{O}_{X}\right)^{-1} \otimes F_{X}^{*}(E)\right) \\
& =R^{\bullet} f_{*}\left(\theta^{p}\left(\Omega_{f}\right)^{-1} \otimes \psi^{p}(E)\right) .
\end{aligned}
$$

Here the first equality uses the fact that $\psi^{p}=F_{Y}^{*}$ in $K_{0}(Y)$. The second equality follows from the fact that the formation of cohomology commutes with flat base change. The third equality is the definition of $\left(F_{*} \mathcal{O}_{X}\right)^{-1}$ in $K_{0}\left(X^{\prime}\right)\left[\frac{1}{p}\right]$ using Lemmata 3.1 and 3.3. The fourth equality is justified by the projection formula in $K_{0}$-theory (see [8, Prop. 7.13]). The fifth equality is just a simplification. Finally, Proposition 3.2 and Proposition 2.6 imply that

$$
F^{*} F_{*} \mathcal{O}_{X}=\operatorname{Gr}\left(F^{*} F_{*} \mathcal{O}_{X}\right)=\tau\left(I / I^{2}\right)=\theta^{p}\left(I / I^{2}\right)=\theta^{p}\left(\Omega_{f}\right)=\theta^{p}\left(L_{f}\right)
$$

as elements of $K_{0}(X)$. This and the fact that $\psi^{p}=F_{X}^{*}$ in $K_{0}(X)$ prove the last equality, and we are done.

\section{Appendix : another formula for the Bott element}

\section{by Bernhard Köck 1}

The object of this appendix is to give a new formula for the Bott element of a smooth morphism. This formula is analogous to the final displayed formula in the main part of this paper and completes a list of miraculous analogies explained in section 5 of [5]; it also streamlines the proof of Theorem 3.1 in section 3 of [5]. It is probably needless to say that this appendix is inspired by the elegant approach to the Adams-Riemann-Roch theorem in positive characteristic developed by Richard Pink and Damian Rössler in the main part of this paper.

We begin by setting up the context. Let $l$ be a prime and let $f: X \rightarrow Y$ be a smooth quasi-projective morphism between Noetherian schemes of relative

\footnotetext{
${ }^{1}$ School of Mathematics, University of Southampton, SO17 1BJ, United Kingdom. e-mail: B.Koeck@soton.ac.uk
} 
dimension $d$. We furthermore assume that there exists an ample invertible $\mathcal{O}_{X^{-}}$ module. Let $\Omega_{f}$ denote the lcoally free sheaf of relative differentials and let $\theta^{l}\left(\Omega_{f}\right) \in K_{0}(X)$ denote the $l$-th Bott element associated with $\Omega_{f}$ (see Introduction). Furthermore let $\Delta: X \rightarrow X^{l}$ denote the diagonal morphism from $X$ into the $l$-fold cartesian product $X^{l}:=X \times_{Y} \ldots \times_{Y} X$. We view $\Delta$ as a $C_{l}$-equivariant morphism where the cyclic group $C_{l}$ of order $l$ acts trivially on $X$ and by permuting the factors on $X^{l}$. In particular we have a pull-back homomorphism $\Delta^{*}: K_{0}\left(C_{l}, X^{l}\right) \rightarrow K_{0}\left(C_{l}, X\right)$ between the corresponding Grothendieck groups of equivariant locally free sheaves on $X^{l}$ and $X$, respectively. As the closed immersion $\Delta$ is also regular we furthermore have a push-forward homomorphism $\Delta_{*}: K_{0}\left(C_{l}, X\right) \rightarrow K_{0}\left(C_{l}, X^{l}\right)$ (see section 3 in $[6]$ ). Let finally $\left(\left[\mathcal{O}_{X}\left[C_{l}\right]\right]\right.$ ) denote the principal ideal of $K_{0}\left(C_{l}, X\right)$ generated by the regular representation $\left[\mathcal{O}_{X}\left[C_{l}\right]\right]$. We have a natural map $K_{0}(X) \rightarrow K_{0}\left(C_{l}, X\right) \rightarrow K_{0}\left(C_{l}, X\right) /\left(\left[\mathcal{O}_{X}\left[C_{l}\right]\right]\right)$ which is in fact injective under certain rather general assumption (see Corollary 4.4 in [5]). The following theorem strengthens Theorem 3.1 in [5]; it should be viewed as an analogue of the formula $\theta^{p}\left(\Omega_{f}\right)=F^{*} F_{*}\left(\mathcal{O}_{X}\right)$ proved at the very end of the main part of this paper.

Theorem. We have

$$
\left.\theta^{l}\left(\Omega_{f}\right)=\Delta^{*}\left(\Delta_{*}\left(\mathcal{O}_{X}\right)\right)\right) \quad \text { in } K_{0}\left(C_{l}, X\right) /\left(\left[\mathcal{O}_{X}\left[C_{l}\right]\right]\right)
$$

Proof. Let $\mathcal{I}_{\Delta}$ denote the ideal sheaf corresponding to the regular closed immersion $\Delta: X \rightarrow X^{l}$. Then we have

$$
\Delta^{*}\left(\Delta_{*}\left(\mathcal{O}_{X}\right)\right)=\lambda_{-1}\left(\mathcal{I}_{\Delta} / \mathcal{I}_{\Delta}^{2}\right) \quad \text { in } K_{0}\left(C_{l}, X\right)
$$

by the equivariant self-intersection formula (see Corollary (3.9) in [6]); here $\lambda_{-1}(\mathcal{E})$ denotes the alternating sum $\left[\mathcal{O}_{X}\right]-[\mathcal{E}]+\left[\Lambda^{2}(\mathcal{E})\right] \pm \ldots$ for any locally free $C_{l^{-}}$sheaf $\mathcal{E}$ on $X$. Furthermore we know that $\mathcal{I}_{\Delta} / \mathcal{I}_{\Delta}^{2}$ is $C_{l^{-}}$-isomorphic to $\Omega_{f} \otimes \mathcal{H}_{X, l}$ where $\mathcal{H}_{X, l}:=\operatorname{ker}\left(\mathcal{O}_{X}\left[C_{l}\right] \stackrel{\text { sum }}{\longrightarrow} \mathcal{O}_{X}\right)$ denotes the augmentation representation (see Lemma 3.5 in [5]). Finally we have $\lambda_{-1}\left(\mathcal{E} \otimes \mathcal{H}_{X, l}\right)=\theta^{l}(\mathcal{E})$ in $K_{0}\left(C_{l}, X\right) /\left(\left[\mathcal{O}_{X}\left[C_{l}\right]\right]\right)$ for any locally free $C_{l}$-module $\mathcal{E}$ on $X$ (see Proposition 3.2 and Remark 3.9 in [5]). Putting these three facts together we obtain the desired equality of classes in $K_{0}\left(C_{l}, X\right) /\left(\left[\mathcal{O}_{X}\left[C_{l}\right]\right]\right)$.

Remarks.

(a) As in the remark after Lemma 3.3 in the main part of this paper, using the projection formula, we can easily derive the original formula $\Delta_{*}\left(\lambda_{-1}\left(\Omega_{f} \otimes \mathcal{H}_{X, l}\right)^{-1}\right)=$ 
1 in $K_{0}\left(C_{l}, X\right)\left[l^{-1}\right] /\left(\left[\mathcal{O}_{X}\left[C_{l}\right]\right]\right)$ (see Theorem 3.1 in [5]) from (the proof of) the above theorem.

(b) The following table summarizes the astounding analogies mentioned at the beginning of this appendix. While the left hand column refers to the situation of the main part of this paper the right hand column refers to the situation of this appendix and of section 4 in [5]. The entries in the table are of a very symbolic nature; more detailed explanations can be found in section 5 of [5]. For instance, $\tau^{l}: K_{0}(X) \rightarrow K_{0}\left(C_{l}, X\right)$ and $\tau_{\text {ext }}^{l}: K_{0}(X) \rightarrow K_{0}\left(C_{l}, X^{l}\right)$ denote the $l$-th tensor-power operation and $l$-th external-tensor-power operation, respectively.

\begin{tabular}{|c||c|}
\hline$\psi^{p}=F_{X}^{*}$ & $\psi^{l}=\tau^{l}$ \\
\hline relative Frobenius $F: X \rightarrow X^{\prime}$ & diagonal $\Delta: X \rightarrow X^{l}$ \\
\hline$f$ is smooth & $f$ is smooth \\
$\Rightarrow F$ is flat & $\Rightarrow \Delta$ is regular \\
$\Rightarrow$ We have $F_{*}: K_{0}(X) \rightarrow K_{0}\left(X^{\prime}\right)$ & $\Rightarrow$ We have $\Delta_{*}: K_{0}\left(C_{l}, X\right) \rightarrow K_{0}\left(C_{l}, X^{l}\right)$ \\
\hline$f^{\prime}: X^{\prime} \rightarrow Y$ & $f^{l}: X^{l} \rightarrow Y$ \\
\hline$J^{*}: K_{0}(X) \rightarrow K_{0}\left(X^{\prime}\right)$ & $\tau_{\text {ext }}^{l}: K_{0}(X) \rightarrow K_{0}\left(C_{l}, X^{l}\right)$ \\
\hline Base change: $F_{Y}^{*} f_{*}=\left(f^{\prime}\right)_{*} J^{*}$ & Künneth formula: $\tau^{l} f_{*}=f_{*}^{l} \tau_{\text {ext }}^{l}$ \\
\hline$F_{X}^{*}=F^{*} J^{*}$ & $\tau^{l}=\Delta^{*} \tau_{\text {ext }}^{l}$ \\
\hline$\theta^{p}\left(\Omega_{f}\right)=F^{*}\left(F_{*}\left(\mathcal{O}_{X}\right)\right)$ & $\theta^{l}\left(\Omega_{f}\right)=\Delta^{*}\left(\Delta_{*}\left(\mathcal{O}_{X}\right)\right)$ \\
\hline
\end{tabular}

\section{References}

[1] Paul Baum, William Fulton, and Robert MacPherson, Riemann-Roch for singular varieties, Inst. Hautes Études Sci. Publ. Math. (1975), no. 45, 101-145.

[2] J.-M. Bismut, H. Gillet, and C. Soulé, Analytic torsion and holomorphic determinant bundles. I. Bott-Chern forms and analytic torsion, Comm. Math. Phys. 115 (1988), no. 1, 49-78.

[3] Barbara Fantechi, Lothar Göttsche, Luc Illusie, Steven L. Kleiman, Nitin Nitsure, and Angelo Vistoli, Fundamental algebraic geometry, Mathematical Surveys and Monographs, vol. 123, American Mathematical Society, Providence, RI, 2005. Grothendieck's FGA explained.

[4] William Fulton and Serge Lang, Riemann-Roch algebra, Grundlehren der Mathematischen Wissenschaften [Fundamental Principles of Mathematical Sciences], vol. 277, SpringerVerlag, New York, 1985.

[5] B. Köck, Riemann-Roch for tensor powers, Math. Z. 233 (2000), no. 4, 755-801. 
[6] Bernhard Köck, The Grothendieck-Riemann-Roch theorem for group scheme actions, Ann. Sci. École Norm. Sup. (4) 31 (1998), no. 3, 415-458 (English, with English and French summaries).

[7] Ernst Kunz, Kähler differentials, Advanced Lectures in Mathematics, Friedr. Vieweg \& Sohn, Braunschweig, 1986.

[8] Ju. I. Manin, Lectures on the K-functor in algebraic geometry, Uspehi Mat. Nauk 24 (1969), no. 5 (149), 3-86 (Russian).

[9] Madhav V. Nori, The Hirzebruch-Riemann-Roch theorem, Michigan Math. J. 48 (2000), 473-482. Dedicated to William Fulton on the occasion of his 60th birthday. 